livraisons

d'Histoire

de l'Architecture

\section{Livraisons de l'histoire de l'architecture}

32 | 2016

Les représentations de l'architecture

\title{
La représentation de la technique en France sous l'Ancien régime
}

The representation of technique in France throughout Ancien Régime

Technikdarstellungen in Frankreich im Ancien Régime

Jason Nguyen

\section{OpenEdition}

Journals

Édition électronique

URL : http://journals.openedition.org/lha/632

DOI : $10.4000 /$ lha. 632

ISSN : 1960-5994

Éditeur

Association Livraisons d'histoire de l'architecture - LHA

Édition imprimée

Date de publication : 31 décembre 2016

Pagination : 21-35

ISSN : 1627-4970

Référence électronique

Jason Nguyen, "La représentation de la technique en France sous l'Ancien régime », Livraisons de I'histoire de l'architecture [En ligne], 32 | 2016, mis en ligne le 31 décembre 2018, consulté le 30 avril 2019. URL : http://journals.openedition.org//ha/632 ; DOI : 10.4000/lha.632 


\section{LA REPRÉSENTATION DE LA TECHNIQUE EN FRANCE SOUS L'ANCIEN RÉGIME}

Une estampe par le graveur François I ${ }^{\text {er }}$ Poilly montre trois dessins attribués à Michel Hasté, maître-serrurier auteur des portes de la Cour d'Honneur (16791681), de l'Orangerie (1681-1687) et du Trianon de Marbre (1687-1688) de Versailles et de la grille de la place Royale de Paris (1682) (ill. 1) ${ }^{1}$. Les formes courbées en fer des deux rampes imitent les balustres classiques, généralement en pierre ou en bois ${ }^{2}$. La ductilité de la matière permet à Hasté de modeler les feuilles d'acanthes à sa guise. Son aspect plat et malléable rendait la représentation du fer particulièrement adaptée à la technique de l'estampe, le lien étant si évident que Poilly a entièrement hachuré de lignes horizontales et verticales les tiges courbes, sans prendre en compte le grain du fer forgé. Ici, la représentation de la matière s'oppose à sa composition interne et au forgeage qui détermine son élasticité.

Cet article analyse la façon dont les architectes, les artisans et les graveurs ont illustré les ouvrages dépendant de l'art de la serrurerie durant les XVII ${ }^{\mathrm{e}}$ et XVIII ${ }^{\mathrm{e}}$ siècles. L'objectif est de comprendre comment une estampe peut illustrer la relation entre la fabrication du fer et sa représentation. L'exemple de Poilly met en évidence les tensions entre le classicisme architectural et les techniques métallurgiques qui lui sont contemporaines. À bien des égards, la ductilité du métal et la relative facilité de sa représentation masquent la complexité du processus de fabrication, seulement sensible dans les ornements employés. Le coq gaulois, la fleur de lys et le soleil rappellent, par leur volume, le caractère politique et écono-

1. Sur cette planche, voir Louis Blanc, Le Fer forgé en France aux XVI et XVII siècles: auvres gravées des anciens maîtres serruriers, architectes, dessinateurs et graveurs, Paris/Bruxelles, V. Van Oest, 1928,

27 p. et Le fer forgé en France. La Régence: Aurore, Apogée, Déclin : auvres gravées des anciens maîtres serruriers, architectes, dessinateurs et graveurs, Paris/Bruxelles, V. Van Oest, 1930, 24 p. Sur le travail de Michel Hasté, voir Alexandre Gady, "Transformation des logis sur cour ", Aurélia Rostaing, "Orangerie " et Raphaël Masson, "Trianon de marbre ", Alexandre Gady dir., Jules HardouinMansart, 1646-1708, Paris, MSH, 2010, p. 171-176, 185-190 et 283-197. Sur la grille à la place Royale (actuellement place des Vosges), voir Isabelle Dérens, "La grille de la place Royale » De la place Royale à la place des Vosges, Béatrice De Andia et Alexandre Gady dir., Paris, Action artistique de la ville de Paris, 1996, p. 74-77.

2. Catherine Arminjon, "Le fer forgé dans les hôtels du Marais ", Le Marais : mythe et réalité, Paris, Picard, 1987, p. 214-220 ; Michel Le Moël, L'Architecture privée à Paris au Grand Siècle, Paris, Commission des travaux historiques de la ville de Paris, 1990, p. 90-97; Marie-France LacoueLabarthe, L'Art du fer forgé en pays bordelais de Louis XIV à la Révolution, Bordeaux, Société Archéologique de Bordeaux, 1993, 367 p. ; Jean-François Leiba-Dotenwill et Roselyn Bussière "Les rampes de serrurerie parisiennes aux XVII ${ }^{\mathrm{e}}$ et XVIII ${ }^{\mathrm{e}}$ siècles ", Escaliers parisiens sous l'Ancien Régime : l'apogée de la serrurerie, Paris, Somogy, 2011, p. 32-47. 


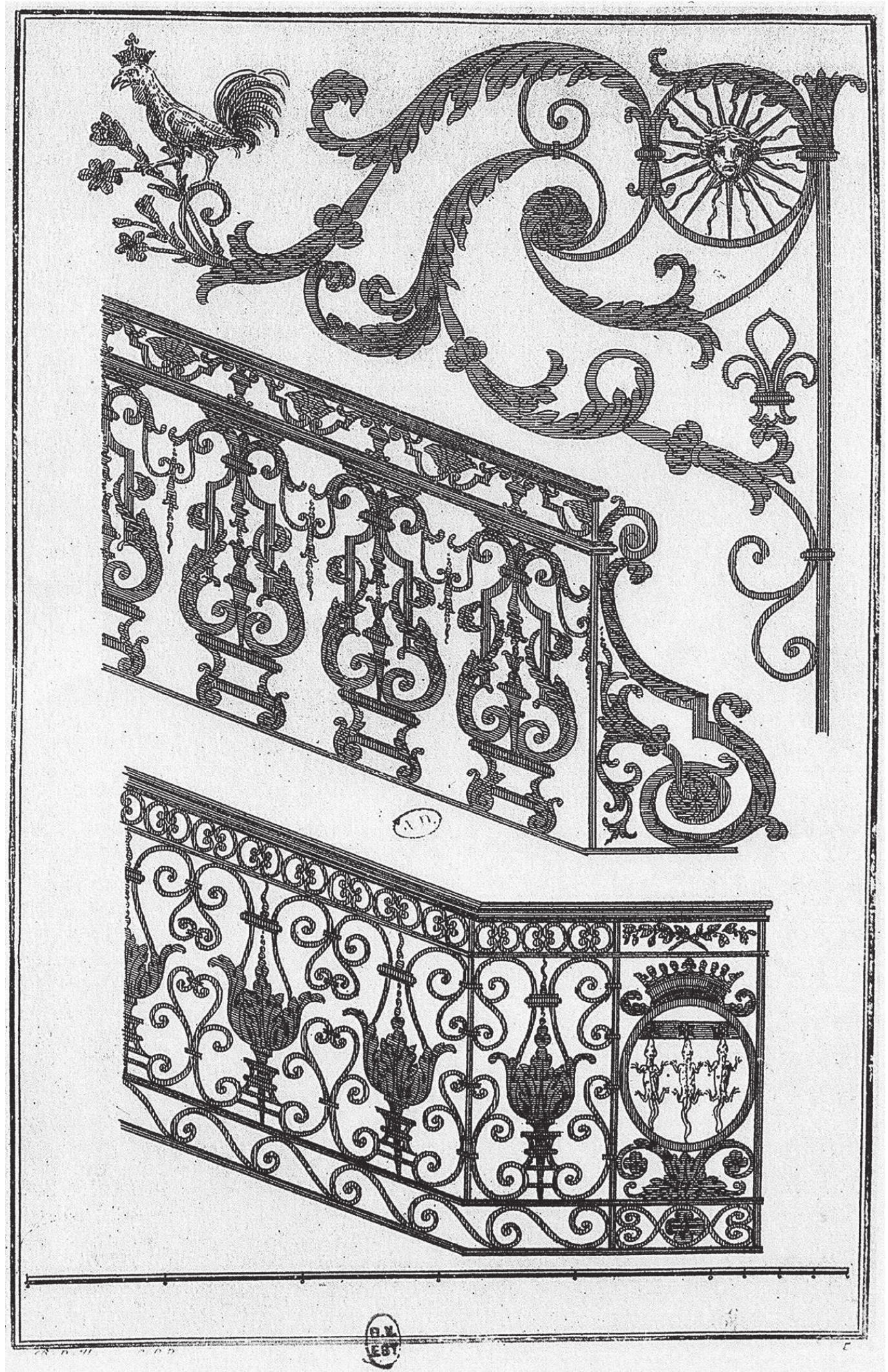

Ill. 1 : «Deux rampes attribué à Michel Hasté », gravure à l'eau-forte, François I ${ }^{\text {er }}$ Poilly, reproduit dans Louis Blanc, Le Fer forgé en France aux XVI et XVII siècles : auvres gravées des anciens maîtres serruriers, architectes, dessinateurs et graveurs, Paris/Bruxelles, V. Van Oest, 1928, pl. 31. () Cl. Jason Nguyen. 
mique de l'industrie métallurgique. On trouve une rampe en fonte similaire à celle représentée au centre de l'estampe à l'hôtel Le Peletier de Saint-Fargeau, achevé en 1687 par l'architecte Pierre Bullet pour Michel Le Peletier de Souzy, intendant des Finances et directeur général des Fortifications sous Louis XIV ${ }^{3}$. Bien que l'implication d'Hasté dans le projet n'est jamais été démontrée, la coexistence de l'illustration et de la rampe atteste de la relation complexe entre l'architecture, la technologie et le marché des estampes.

L'utilisation accrue de la serrurerie pendant la période est concomitante de l'essor de l'industrie métallurgique en Europe et de l'influence croissante du marché des estampes. Le fer était l'une des matières premières les plus importantes de la période moderne et de nombreuses guerres furent menées pour le contrôle de territoires riches en ressources naturelles, les politiques commerciales visant à garder le minerai à l'intérieur des frontières nationales ${ }^{4}$. À court terme, ce protectionnisme alimentait l'effort de guerre, au sens propre comme au sens figuré. En France, la production de l'artillerie pour l'armée royale et des ancres pour la marine constituait le pilier de l'industrie métallurgique, et la serrurerie une de ses nombreuses branches. Sur l'estampe de Poilly, le graveur incorpore d'ailleurs trois lézards dans la rampe du bas, référence aux armoiries familiales de Michel Le Tellier, Marquis de Louvois. De dernier détenait la charge de secrétaire d'État à la Guerre depuis 1662 et, à partir de 1682 jusqu'à sa mort en 1691, celle de surintendant des Bâtiments, Arts et Manufactures de France. Au cours de son exercice, l'idéologie militaire et l'architecture firent progressivement partie de la même sphère d'influence 5 .

Le présent article s'articule autour de questions liées à la représentation de l'art de serrurerie sous l'Ancien Régime. Comment les estampes mettent en évidence ou bien masquent les réalités matérielles et les techniques du fer, et de quelles façons cette technique de l'estampe a-t-elle influencé les formes que le métal prendra par la suite? Quelles sont les raisons et les conséquences de l'intérêt renouvelé des

3. Hubert Beylier et Daniel Bontemps, «La rampe en fonte du XVII siècle de l'escalier de l'hôtel Le Peletier de Saint-Fargeau à Paris ", Bulletin de la Société de l'histoire de Paris et de l'Île-de-France, vol. 110-111, 1984, p. 123-131. Charles Sellier a longtemps daté la rampe au XIX siècle. Charles Sellier, Hôtel de Saint-Fargeau à Paris, Saint-Denis, Impr. H. Bouillant, 1895, 23 p. et Anciens hôtels de Paris, Paris, H. Champion, 1910, p. 2.

4. Pour mieux comprendre les politiques commerciales du fer en France et ailleurs en Europe, voir Bertrand Gille, Les Origines de la grande industrie métallurgique en France, Paris, DomatMontchrestien, 1947, 212 p. ; Abraham Cronstrom, Iron and Steel on the European Market in the 17th Century: A Contemporary Swedish Account of the Production Forms and Marketing, Stockholm, Historical Metallurgy Group of the Swedish Ironmasters' Association, 1982, 272 p. ; Jean-François Belhoste et al. dir., La Métallurgie normande, XII ${ }^{e}$-XVII ${ }^{e}$ siècles : la révolution du haut fourneau, Caen, AHPIN, 1991, 322 p. et surtout "L'âge classique : deuxième moitié XVII"-XVIII" siècles ", La Métallurgie comtoise, XVe-XIX siècles: Étude du Val de Saône, Besançon, SPADEM, 1994, p. 119-255 ; Hjalmar Fors, The Limits of Matter: Chemistry, Mining, and the Enlightenment, Chicago, The University of Chicago Press, 2015, 241 p.

5. Sur la relation entre l'architecture et la guerre sous Louvois, voir Thierry Sarmant, Les Demeures du Soleil: Louis XIV, Louvois et la surintendance du Bâtiment du Roi, Seyssel, Champ Vallon, 2003, 387 p. 
architectes, artisans et graveurs pour les techniques de métallurgie au tournant $\mathrm{du}$ $\mathrm{XVIII}^{\mathrm{e}}$ siècle ? Et quel était le rôle joué par le marché des estampes dans la représentation et la transmission de cette technique?

\section{La représentation de l'artisanat}

L'artisanat et la connaissance du matériau jouèrent un rôle essentiel dans la représentation de la serrurerie au début du XVII ${ }^{\mathrm{e}}$ siècle. Dans La fidelle ouverture de l'art de serrurerie publiée en 1627, Mathurin Jousse, maître-serrurier et théoricien de l'architecture, explique comment l'extraction et la fabrication du fer influencent sa couleur et sa ductilité ${ }^{6}$. Première œuvre en français à mettre en texte et en image l'expertise du serrurier, son traité représente les formes complexes qu'il est possible d'obtenir par la modélisation de la matière (ill. 2) ${ }^{7}$. En décrivant les clés figurées dans la première planche, il accorde autant d'importance à la complexité du panneton qu'à son ornementation, en soulignant l'utilisation de motifs architecturaux comme les chapiteaux de colonne, les balustres, les feuillages et les grotesques ${ }^{8}$. Le fait qu'il explique et illustre plusieurs outils et machines nécessaires à la fabrication du fer témoigne de l'ampleur des compétences techniques du maître-serrurier ${ }^{9}$.

Le traité de Jousse est important car il présente les premières étapes pour comprendre la relation entre la connaissance des matériaux et la création artistique. Par la suite, les serruriers ont souvent utilisé les estampes pour faire abstraction de la technicité que Jousse mettait en avant dans son traité. Prenons l'exemple du Livre de Serrurerie que Robert Davesne a publié en 1676 dans son atelier rue Neuve-

6. Mathurin Jousse, La fidelle ouverture de l'art de serrurier, La Flèche, Chez Georges Griveau, 1627, p. 138. Jousse a aussi publié des traités sur la stéréotomie et la charpenterie ainsi qu'une édition du traité sur la perspective par Viator. Voir Carmen Pérez de los Ríos et Elena García Alías, "Marthurin Jousse, preludio de la estereotomía moderna ", Actas del Sexto Congreso Nacional de Historia de la Construcción 10, 2009, p. 1041-1050 ; Émile-C. Pequet, "Mathurin Jousse, architecte et ingénieur de la ville de la Flèche au XVII e siècle ", Cahiers Fléchois 6, 1984, p. 28-41.

7. Le premier traité sur l'industrie métallurgique a été rédigé par Georgius Agricola, De re metallica, Paris, H. Gormontium, 1556, in- $8^{\circ}$.

8. Mathurin Jousse, La fidelle ouverture de l'art de serrurier, op. cit., p. 10-11.

9. Sur les serruriers et les corps des métiers, voir René de Lespinasse, Les Métiers et corporations de la Ville de Paris XIV'e-XVIII e siècles, Paris, Imp. nationale, 1879-1897, 3 vol. ; François Olivier-Martin, L'Organisation corporative de la France d'Ancien Régime, Paris, Sirey, 1938, 565 p. ; Émile Coornaert, Les corporations en France avant 1789, Paris, Gallimard, 1968 ( $2^{\mathrm{e}}$ éd.), 316 p. ; William Sewell, Work and Revolution in France: The Language of Labor from the Old Regime to 1848, Cambridge, Cambridge University Press, 1980, p. 16-39; Anthony Black, Guilds and civil society in European political thought from the twelfth century to the present, London, Mehuen, 1984, p. 123-156; JeanLouis Bourgeon, "Colbert et les corporations : l'exemple de Paris ", Un Nouveau Colbert: Actes du Colloque pour le tricentenaire de la mort de Colbert, Jean Favier et Roland Mousnier dir., Paris, éd. SEDES/CDU, 1985, p. 241-253. 


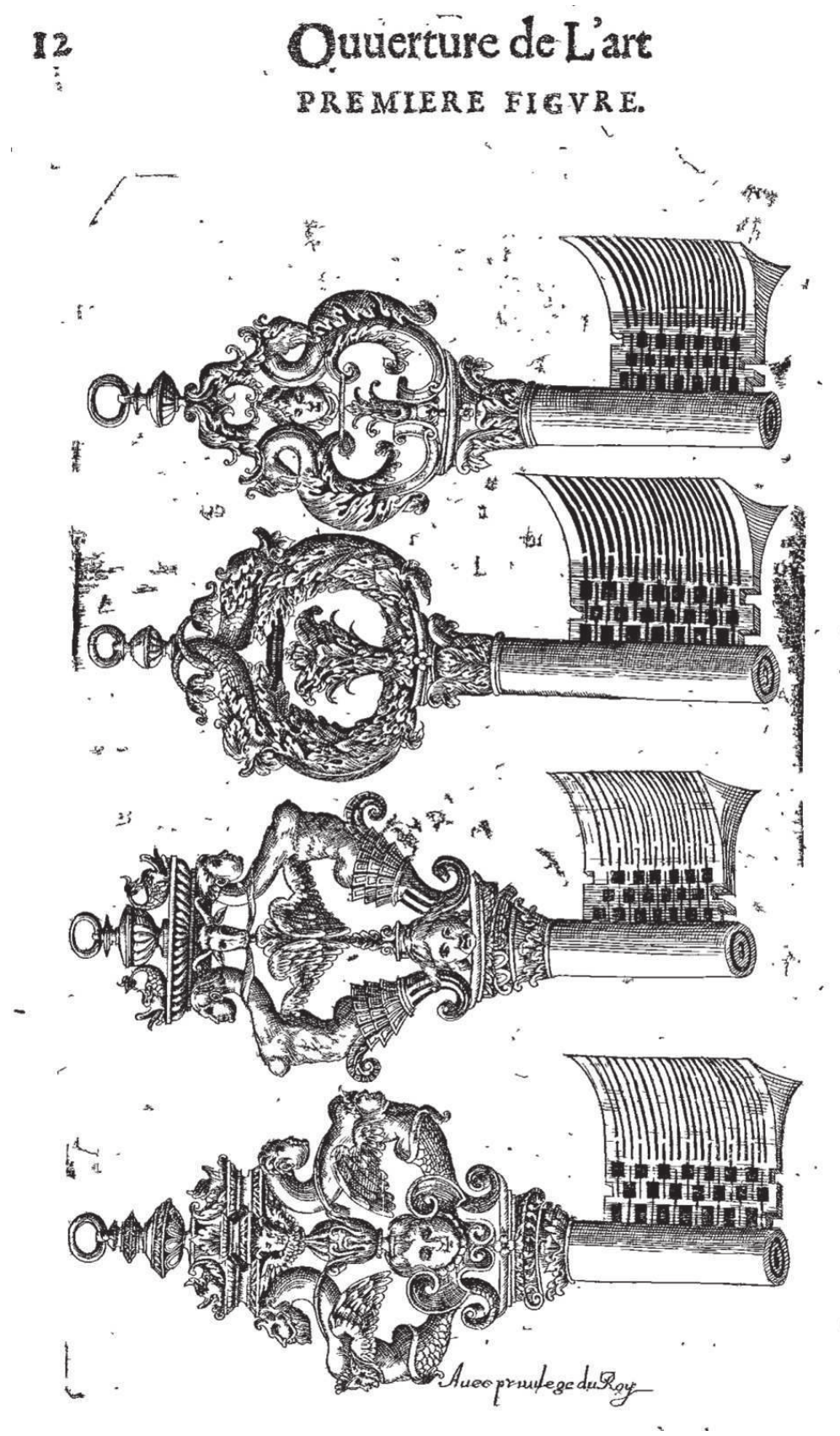

Ill. 2 : "Les clefs ", gravure à l'eau-forte, Mathurin Jousse, La Fidelle ouverture de l'art de serrurerie, La Flèche, Chez Georges Griveau, pl. 1. (C) Cl. Bibliothèque nationale de France.

Montmartre (ill. 3) ${ }^{10}$. Vêtu comme un bourgeois, le serrurier est assis à une table sur laquelle sont disposés les outils du graveur; un jardin d'une propriété royale s'étendant à l'arrière-plan. La relation dialectique entre la nature et l'art est introduite par le va-et-vient visuel entre les formes des parterres à l'arrière et les barreaux en fer forgé. Ceux-ci montrent des options décoratives différentes, qui illustrent la

10. Robert Davesne, Livre de Serrurerie nouvellement inventé par Robert Davesne, Paris, Chez l'Auteur, 1676 , in- $4^{\circ}, 3 \mathrm{ff}$. 


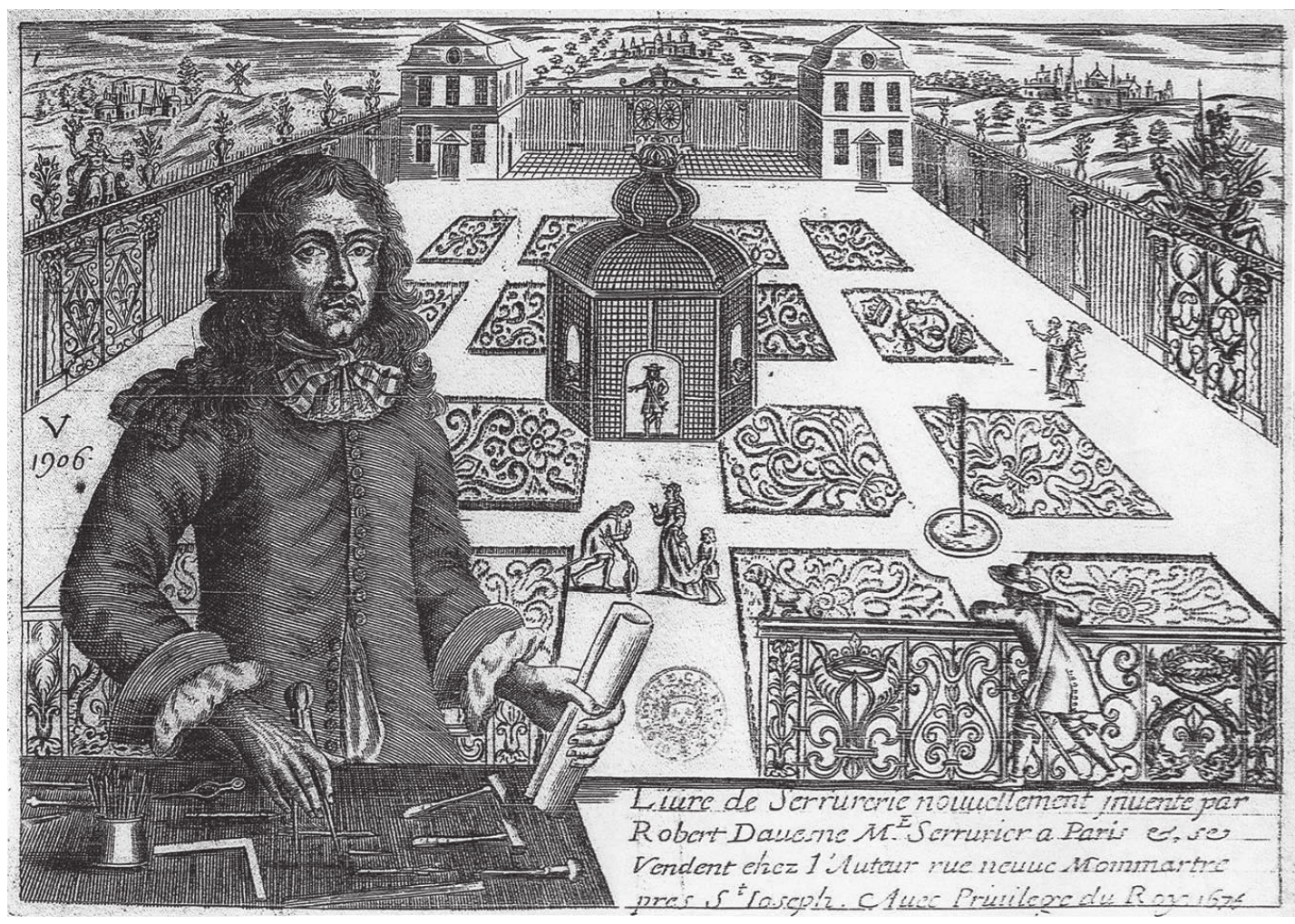

Ill. 3 : "Robert Davesne, Livre de serrurerie nouvellement inventé par Robert Davesne (1676)", gravure à l'eau-forte, Robert Davesne, Livre de serrurerie nouvellement inventé par Robert Davesne, Paris, Chez l'auteur, 1676, frontispice. (C) Cl. Jason Nguyen.

variété possible des formes grâce à la malléabilité du fer et, avant tout, à l'esprit inventif de l'artiste.

En France, ce phénomène découle des préoccupations professionnelles des maîtres-serruriers, liées à leurs ambitions sociales, et qui se considéraient plutôt comme des artistes que comme des artisans ${ }^{11}$. Les serruriers collaboraient ou gravaient eux-mêmes ces estampes, tirées de leurs commissions, afin qu'elles soient reproduites par des ouvriers moins adroits ${ }^{12}$. Davesne le souligne en termes explicites, en écrivant aux lecteurs que " tu pourra imiter ou faire imiter par ceux qui seront moins experts que toy ${ }^{13}$. Ce genre de recueil fut bénéfique pour ces deux corps de métier, étant donné qu'ils fournissaient un emploi stable pour les graveurs et augmentaient la visibilité des modèles des serruriers, notamment à la lumière des dimensions délicates de la serrurerie décorative ${ }^{14}$. Un bon exemple de cette

11. L'édit de Saint-Jean-de-Luz (1660) a accordé les privilèges des arts libéraux aux graveurs. Marianne Grivel, Le Commerce de l'estampe à Paris au XVII siècle, Genève, Librairie Droz, 1986, p. 96-99.

12. Jean-François Leiba-Dotenwill et Roselyn Bussière, «Les rampes de serrurerie parisiennes aux XVII et XVIII" siècles ", op. cit., p. 38.

13. Robert Davesne, Livre de Serrurerie, op. cit.

14. Plusieurs auteurs des recueils de l'architecture ont omis la serrurerie, par exemple, dans le cas de la place des Victoires Pierre-Jean Mariette, Architecture françoise, Paris, Chez l'Auteur, t. 1, 1738 et le dessin de la place par Jean-Michel Chevotet (vers 1725), Musée Carnavalet, D 9813. 


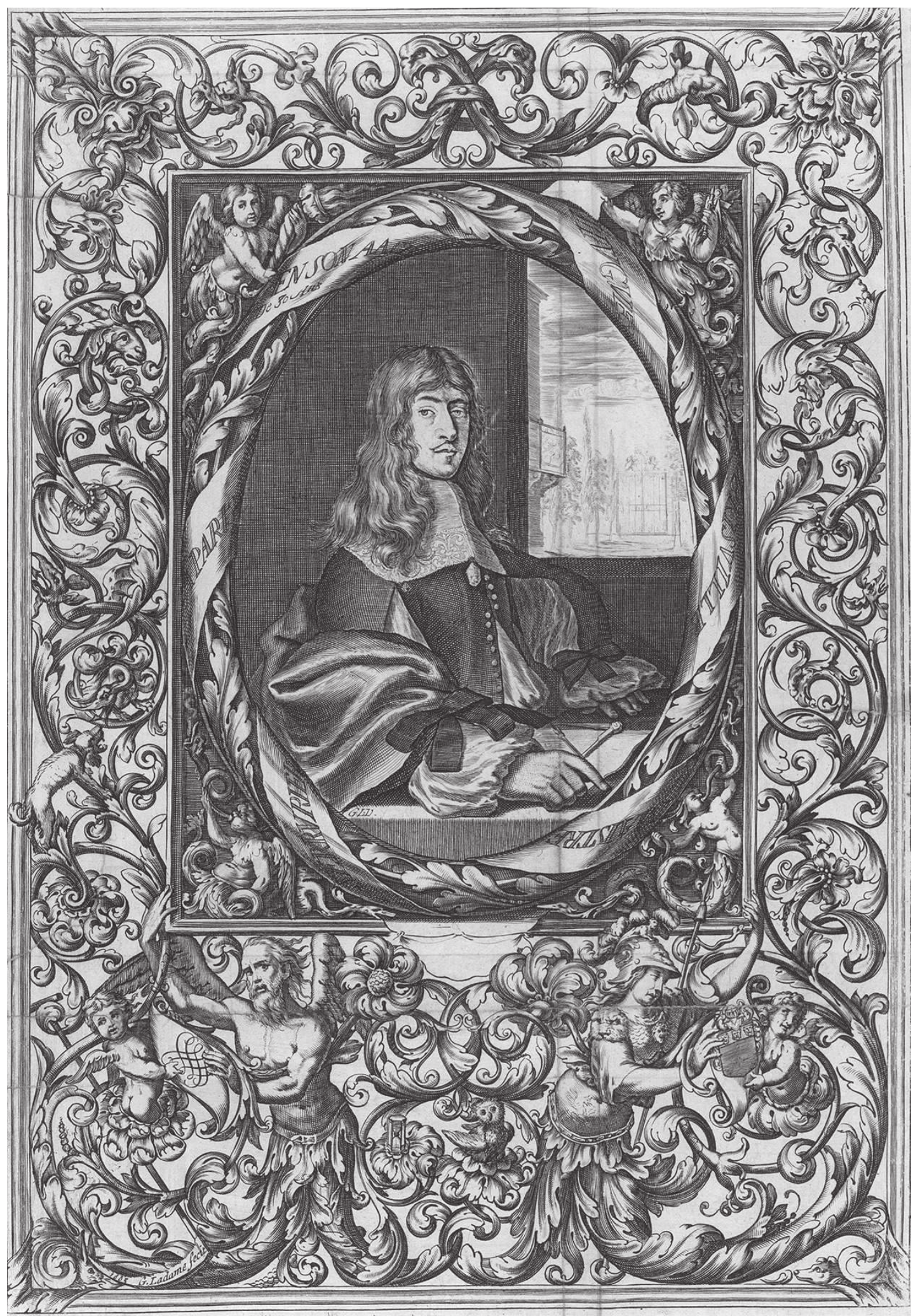

Ill. 4 : «Hughes Brisville ", gravure à l'eau-forte, Gabriel Ladame dans Hughes Brisville, Diverses pièces de serrurerie inventées par Hughes Brisville et gravés par Jean Bérain, Paris, François Langlois, 1663, portrait, p. $2 \mathrm{v}^{\mathrm{o}}$. () Cl. Metropolitan Museum of Art. 
collaboration professionnelle se trouve être les Diverses pièces de serrurerie (1663) par Hughes Brisville et les graveurs Jean Bérain et Gabriel Ladame. Ici, il n'est pas étonnant que le portrait gravé pour cette publication souligne le statut social de l'artisan, et que son nom, gravé sur une écharpe enroulé autour d'un bandeau de feuillage, lise : "Hughes Brisville maistre serrurier à Paris en son age de 30 ans " 15 (ill. 4).

Il a été souligné par les historiens de l'art que le marché des estampes, dont l'influence a augmenté pendant le XVII et le XVIII ${ }^{\mathrm{e}}$ siècle, rendit possible la circulation des modèles entre Paris, Versailles, Londres, Amsterdam et au-delà ${ }^{16}$. Les estampes étaient une forme particulièrement appropriée pour diffuser les inventions artistiques en fer, contrairement à celles de la maçonnerie, qui nécessitaient la mâ̂trise de la tectonique, la logique formelle de la serrurerie pouvant être exprimée en deux dimensions. L'autonomie visuelle de l'objet ornemental dessiné ou imprimé, libérée des contraintes matérielles de la chose qu'il représente, facilite le développement de créations fluides au début du XVIII siècle, telle que la ferronnerie d'art du serrurier lorrain Jean Lamour (ill. 5) ${ }^{17}$.

Le marché des estampes permet un brassage d'informations artistiques, transcendant les barrières de classe, et créant un échange complexe entre, d'une part, la cour à Versailles, et d'autre part, la vie commerciale de Paris ${ }^{18}$. Les Lepautre, une famille d'architectes, de décorateurs et de graveurs, ont travaillé sur les deux marchés et ont publié des recueils en parallèle à leurs projets pour le Cabinet du roi ${ }^{19}$. Prenons l'exemple des estampes de la grille décorative de la grotte de Téthys par Jean Lepautre (ill. 6). André Félibien, théoricien de l'art et historiographe du roi, les incorpore dans ses Descriptions de la grotte de Versailles de 1676 comme illustrations, en affirmant qu' "il n'y a point d'endroit dans toute cette Royale Maison, où l'Art ait réussi plus heureusement ". Il admire la manière dont, dans la faible lueur du soleil couchant, les reflets du métal de la serrurerie "paraissent de véri-

15. Bibliothèque nationale de France, Département des Estampes et de la photographie, RESERVE FOL-QB-201 (50).

16. Marie-France Lacoue-Labarthe, L'Art du fer forgé en pays bordelais de Louis XIV à la Révolution, op. cit., p. 28-45.

17. Jean Lamour, Recueil des ouvrages en serrurerie, que Stanislas le Bien-Faisant, Roy de Pologne, Duc de Lorraine et de Bar, a fait poser sur la place Royale de Nancy, à la gloire de Louis le Bien-Aimé, Nancy, Chez l'Auteur, 1767, [11] p.-28 p. de pl.

18. Fiske Kimball, The Creation of the Rococo, Philadelphia, Philadelphia Museum of Art, 1943, 242 p. ; Bruno Pons, De Paris à Versailles : les sculpteurs ornemanistes parisiens et l'art décoratif des Bâtiments du roi, Strasbourg, Association des publications près les Universités de Strasbourg, 1986, 367 p. ; Katie Scott, The Rococo Interior: Decoration and Social Space in Early Eighteenth-Century Paris, New Haven, Yale University Press, 1995, p. 241-265.

19. Sur l'œuvre des Lepautre, voir Maxime Préaud, Inventaire du fonds français, Graveurs du XVII siècle : Antoine Lepautre, Jacques Lepautre et Jean Lepautre (première partie), Paris, Bibliothèque nationale de France, t. 11, 1993, 369 p. ; Inventaire du fonds français, Graveurs du XVII siècle : Jean Lepautre (deuxième partie), Paris, Bibliothèque nationale de France, t. 12, 1999, 335 p. ; Inventaire du fonds français, Graveurs du XVII e siècle: Pierre Lepautre, Paris, Bibliothèque nationale de France, t. 13, 2008, 382 p. 


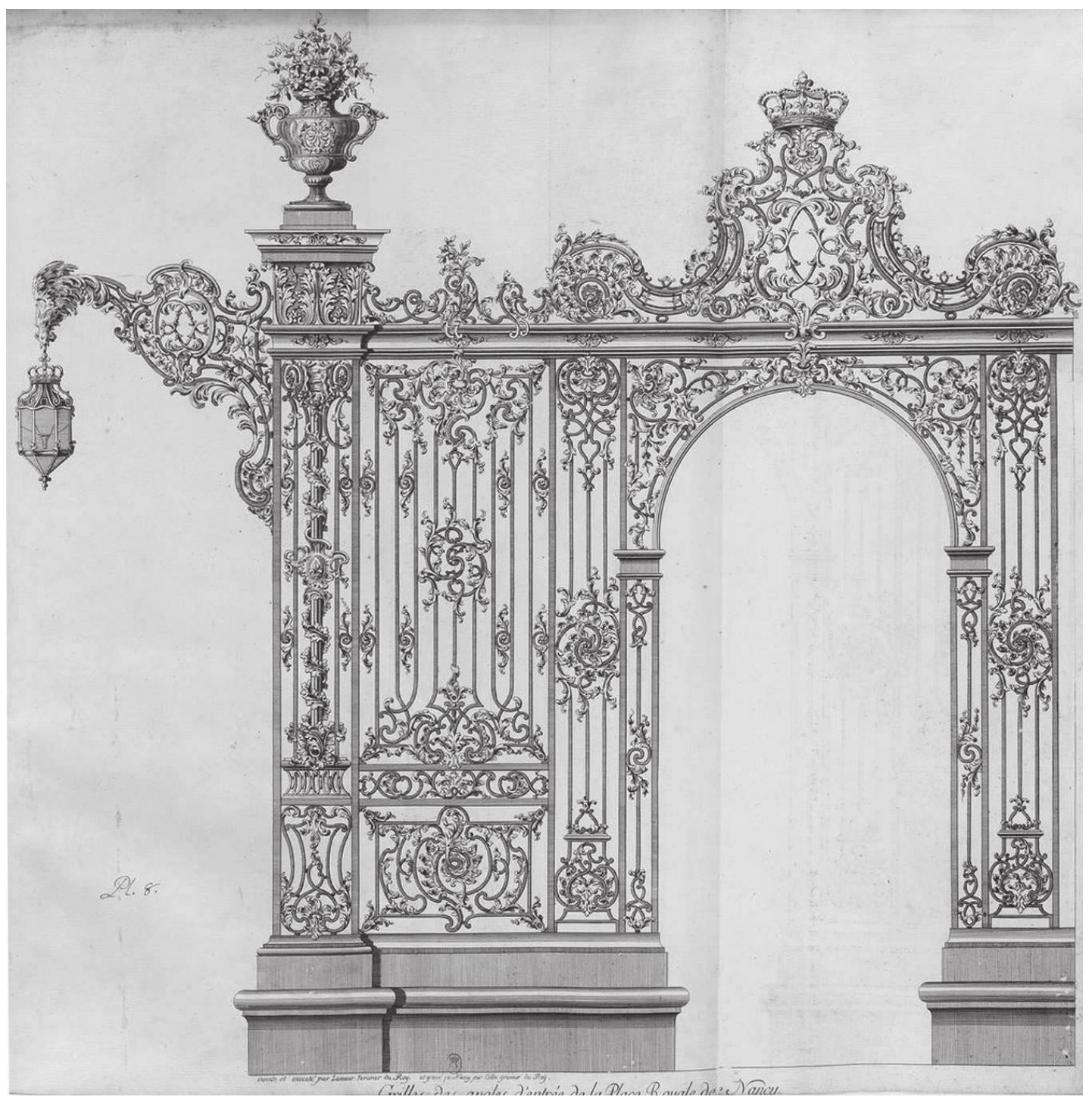

Ill. 5 : "Grilles des angles d'entrée de la place Royale à Nancy", gravure à l'eau-forte, Jean Lamour, Recueil des ouvrages en serrurerie, que Stanislas le Bien-Faisant, Roy de Pologne, Duc de Lorraine et de Bar, a fait poser sur la place Royale de Nancy, à la gloire de Louis le Bien-Aimé, Nancy, Chez l'Auteur, 1767, pl. 11. (C) Cl. Bibliothèque nationale de France.

tables traits de lumière ${ }^{20}$. Cette tournure de phrase, qui traduit l'allégorie du roi Soleil dans le phénomène naturel, aligne l'art et la politique et révèle l'émerveillement lié à la magie du fer reflétant la gloire du règne de Louis XIV ${ }^{21}$. Une fois diffusés, ces exemples suscitent l'émulation parmi la noblesse et une classe de plus en plus influente de financiers qui recherchent alors des modèles similaires - et, dans ce contexte, il n'est pas anodin que la première ait détenu et géré plus de la moitié des mines et des forges de France ${ }^{22}$.

20. André Félibien, Description de la grotte de Versailles, Paris, Imprimerie royale, 1676, p. 1-2.

21. Louis Marin, Le Portrait du roi, Paris, Éd. de Minuit, 1981, 300 p.

22. Guy Chaussinand-Nogaret, La Noblesse au XVIII' siècle : de la féodalité aux Lumières, Bruxelles, Éd. Complexe, 1984, p. 147-150. 


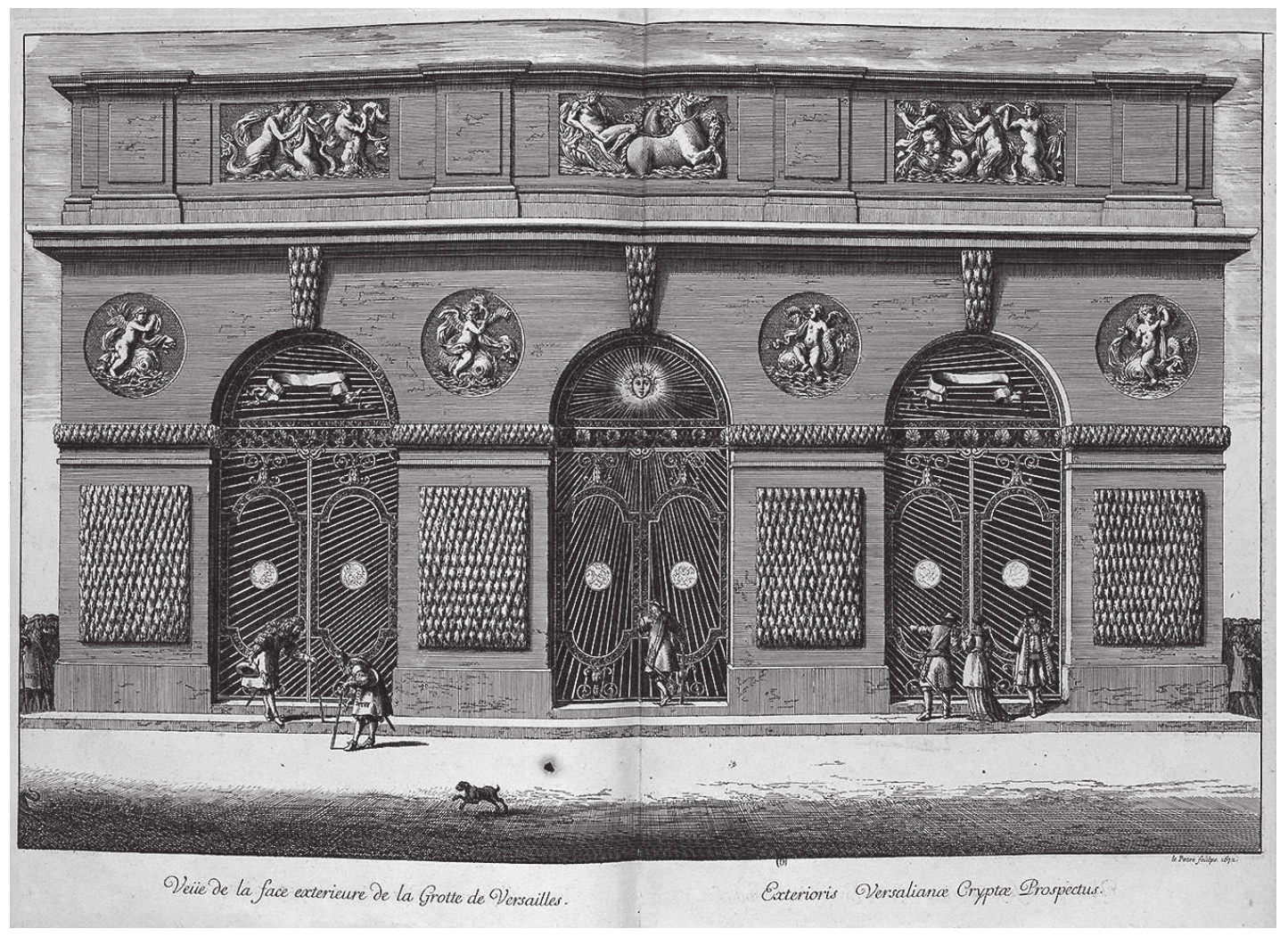

Ill. 6 : «Veüe de la face extérieure de la Grotte de Versailles", gravure à l'eau-forte, Jean Lepautre dans André Félibien, Description de la grotte de Versailles, Paris, Imprimerie royale, 1676, pl. 2. () Cl. Institut nationale d'histoire de l'art.

Dans ces exemples, la représentation idéalisée permet au motif décoratif d'occuper une fonction purement symbolique, en glorifiant son commanditaire en même temps qu'il dissimule les conditions de sa production. Ce contraste illustre le conflit entre la représentation et la technique. Paradoxalement, la facilité par laquelle l'objet en fer forgé peut être traduit en estampe dérive de la malléabilité du matériel, qui lui-même dépend de la qualité du minerai et des techniques de la forge. Cette manufacture d'excellence, en d'autres termes, invite à la dissimulation de la maîtrise artisanale dans la représentation de la décoration en serrurerie.

\section{La représentation de la technique}

L'accent sur la représentation des formes artistique en fer est à mettre en rapport avec trois phénomènes historiques. Premièrement, il a coïncidé avec une période d'augmentation de la production de fer, lié à l'effort de guerre et aux politiques commerciales qui la soutiennent ${ }^{23}$. Deuxièmement, il correspond aux exigences artistiques et techniques qui accompagnent l'aménagement de Versailles, dont l'expansion a commencé pendant les années 1660 et s'est intensifiée avec la

23. Bertrand Gille, Les Origines de la grande industrie métallurgique en France, op. cit., p. 33-54. 
nomination de Jules Hardouin-Mansart comme premier architecte du roi ${ }^{24}$. Par exemple, le montant des dépenses en fer et autres matériaux pour le décor et la dorure du château a atteint en 1685 la somme de 104000 livres $^{25}$. Troisièmement, l'influence croissante du marché des estampes coïncide avec l'établissement de l'Académie royale d'architecture, qui tenta d'élaborer les règles du classicisme architectural. Les traités et les recueils illustrèrent les positions académiques et aidèrent à les diffuser de façon commerciale.

On en trouve un exemple dans le Cours d'architecture suivant les ordres de Vignole publié en 1691 par Augustin-Charles d'Aviler, dans lequel les images sont employées pour traduire la théorie et le savoir-faire ${ }^{26}$. Une des doubles pages $\mathrm{du}$ traité gravée par Jean Dolivar, neveu et élève de Jean Lepautre, souligne que le fer pouvait imiter les formes spécifiquement fabriquées en maçonnerie et en charpenterie. Elle montre aussi les autres options possibles, toutes illustrées sur une même page, rendues possibles grâce aux différentes technologies de fonderie et de montage (ill. 7) ${ }^{27}$. Les balustres désignés par la lettre $l$ indiquent les différents effets réalisables en fer forgé, pendant que celles marquées par un $m$ illustrent les possibilités en fonte ${ }^{28}$. Cette tendance se répète au centre de la planche, qui comprend également des exemples en tôle.

Contrairement à beaucoup de ses prédécesseurs immédiat, D’Aviler se concentre plus sur le rapport entre la fabrication et la forme, rapport qu'il véhicule à travers ces illustrations et un lien avec l'artisanat et qu'il définit ainsi : "Le meilleur moyen pour parvenir à la connoissance des Materiaux, c'est de les travailler : parce qu'il est constaté que personne ne conoist mieux la pierre que celui qui la taille, ni le fer que celui qui le forge ; mais comme chacun ne peut pas estre Ouvrier de profession, on peut néanmoins acquerir cette connoissance par les recherches qu'on en fait $"{ }^{29}$. Son livre, donc, replace la compétence technique au sein de la représentation du classicisme architectural, pour des lecteurs composés à la fois d'artistes libéraux et mécaniques ${ }^{30}$.

Le traité de D'Aviler préfigure l'intérêt des Lumières pour la connaissance technique ainsi que les inquiétudes liées au ralentissement de la production en France

24. Frédéric Tiberghien, Versailles, le chantier de Louis XIV, 1662-1715, Paris, Perrin, 2006, p. 180227.

25. Jules Guiffrey éd., Comptes des Bâtiments du roi sous le règne de Louis XIV, Paris, Imprimerie nationale, t. 2, 1887, p. 613-614, 629.

26. Augustin-Charles D'Aviler, Cours d'architecture qui comprend les ordres de Vignole, Paris, Chez Nicolas Langlois, 1691, 359 p.

27. Ibid., p. 219.

28. Sur les techniques de fabrication du fer fondu, voir "Fer fondu » dans Augustin-Charles D'Aviler, Explication des termes d'architecture, Paris, Chez Nicolas Langlois, 1691, p. 578.

29. Augustin-Charles D'Aviler, Cours, op. cit., p. 201.

30. De juillet à août 1683, quand il travaillait dans l'atelier royal de Jules Hardouin-Mansart, il a présenté les premières ébauches de son traité, en notant que la géométrie simplifiée des ordres de Vignole est "d'un très grand soulagement pour les ouvriers, si elles estoient partout également correctes ". Henry Lemonnier éd., Procès-verbaux de l'Académie royale d'architecture (1671-1793), Paris, Jean Schemit, Librarie de la Société de l'histoire de l'art français, t. 2, 1911-1929, p. 35. 


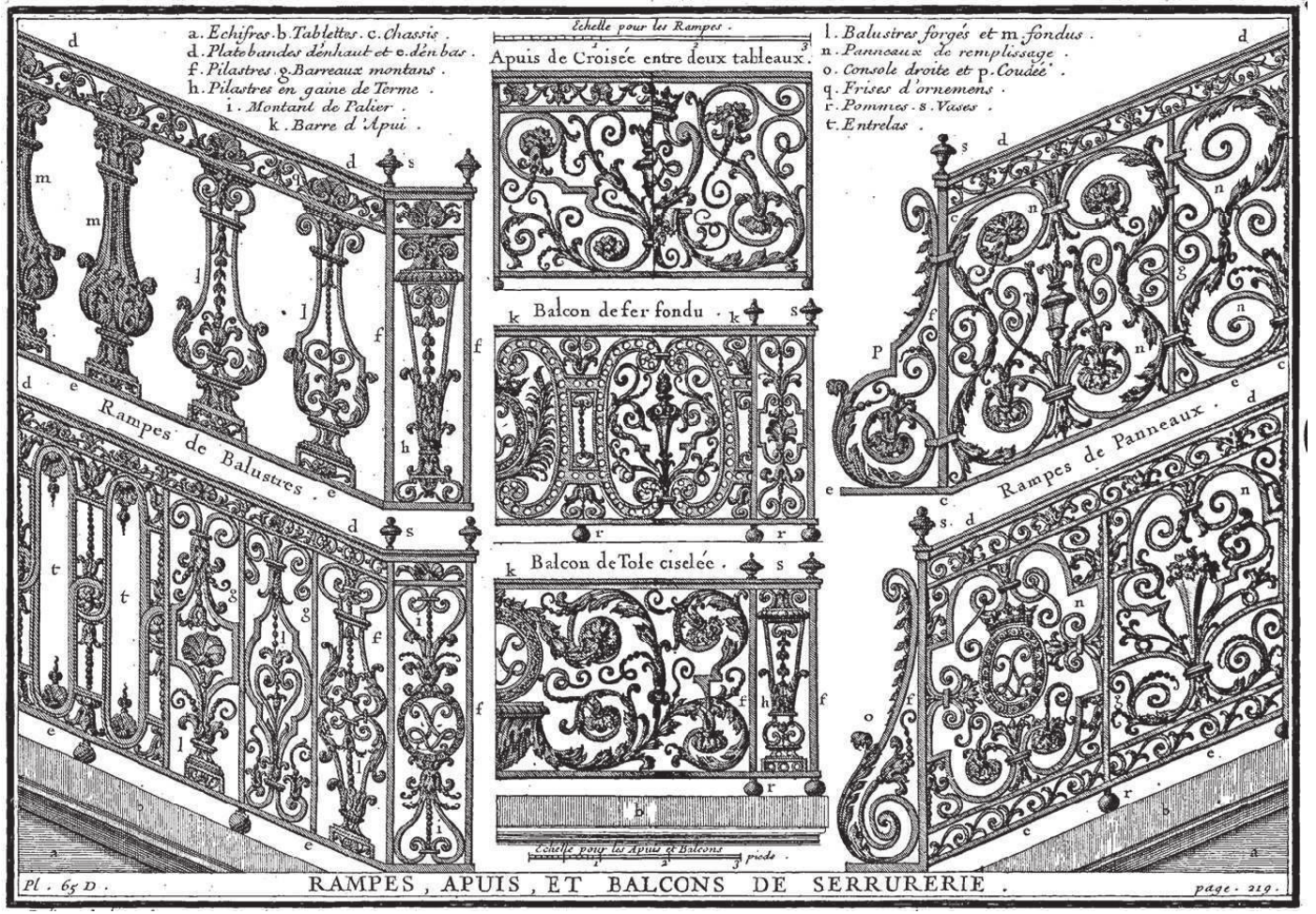

Ill. 7 : «Rampes, appuis, et balcons de serrurerie", gravure à l'eau-forte, Jean Dolivar dans AugustinCharles D'Aviler, Cours d'architecture qui comprend les ordres de Vignole, Paris, Chez Nicolas Langlois, 1691, pl. 65D. (c) Cl. Bibliothèque nationale de France.

vers $1700^{31}$. L'industrie métallurgique française avait souffert dans de nombreuses régions du royaume, en raison des rythmes intenables d'extraction du minerai et de déforestation à partir de 1660 , concomitant à une période de construction intense à Versailles ${ }^{32}$. De plus, la révocation de l'édit de Nantes en 1685 entraine l'exode à l'étranger des artistes et artisans protestants, comme le graveur Daniel Marot et le serrurier Jean Tijou, qui se trouvent tous deux employés par la cour d'Angleterre. Anthony Blunt a qualifié cette période de "déclin de Louis XIV", dont le début a été marqué par l'ascension de Louvois et d'Hardouin-Mansart, ainsi que l'agressivité belliqueuse croissante du roi, focalisé sur la gloire ${ }^{33}$. Les conséquences néfastes politiques et économiques qui ont frappé la France après les guerres de la Ligue d'Augsbourg (1688-1697) et de Succession d'Espagne (17011714) ont provoqué des réductions drastiques des dépenses dans tous les secteurs et la déstabilisation d'une industrie toujours fragile ${ }^{34}$.

31. Bernard Wodon, "La technique du fer forgé au XVIII e siècle. De l'élaboration du fer marchand à l'exécution de la grille ", Bulletin de l'Institut archéologique liégeois, Liège, Maison Curtius, vol. 87, 1975 , p. 31-67.

32. Bertrand Gille, Les Origines de la grande industrie métallurgique en France, op. cit., p. 33-54.

33. Anthony Blunt, "The Decline of Louis XIV, 1685-1701 ", Art and Architecture in France, 15001700, New Haven, Yale University Press, 1999 (5 éd.), p. 241-274.

34. Entre 1685 et 1691 le budget des Bâtiments du roi chute de $87 \%$ (de 15000000 livres en 1685 à 1900000 livres en 1691 - un chiffre resté inchangé jusqu'au début du XVIII siècle). Jules Guiffrey éd., Comptes des Bâtiments du roi, op. cit., t. 2, p. 575-599; t. 3, p. 512-516 ; t. 5, p. 575-579. 


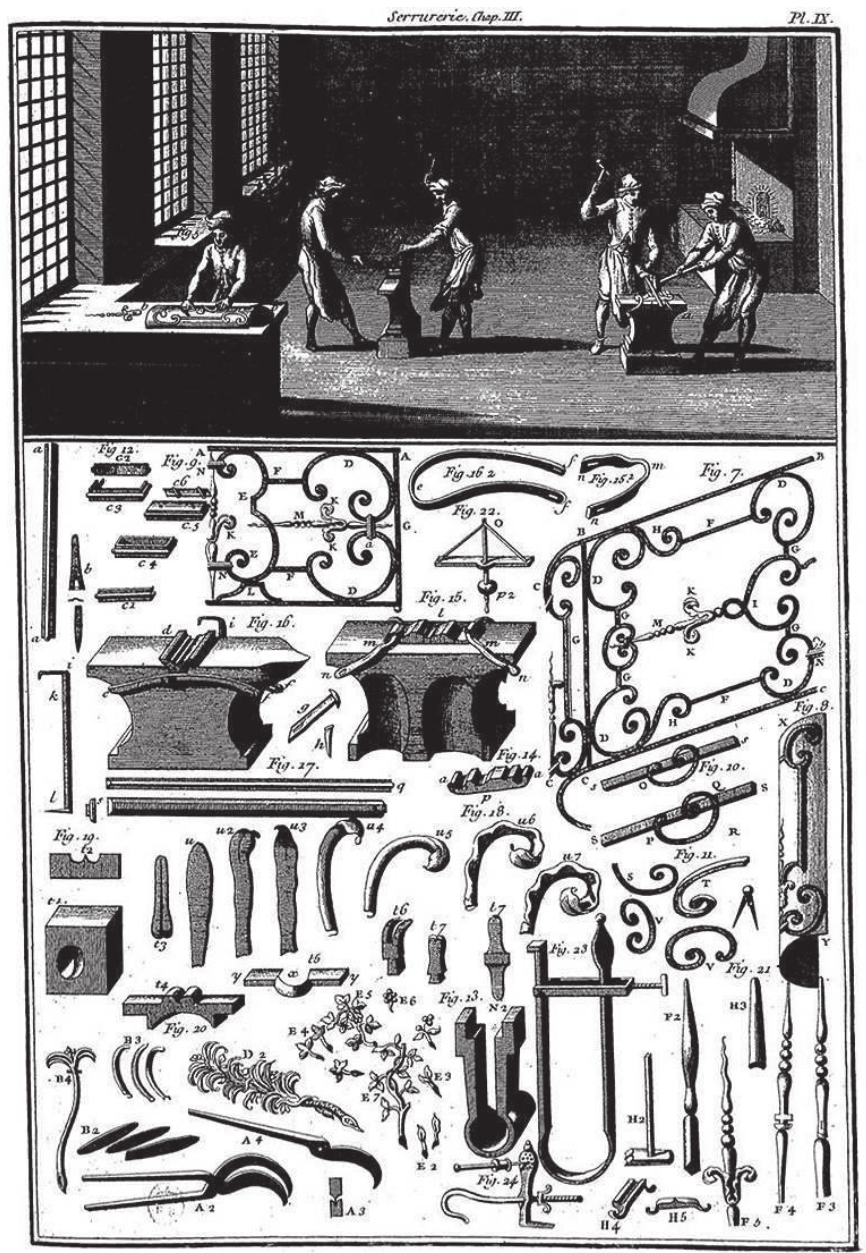

Ill. 8 : «Les outils d'un serrurier ", gravure à l'eau-forte, Henri-Louis Duhamel du Monceau, $A r t d u$ serrurier, Paris, Chez Saillant \& Nyon et chez Desaint, 1757, pl. 9. () Cl. Bibliothèque nationale de France.

Compte tenu de la situation économique de la période, l'accent mis sur l'ouvrier et la représentation de la technique servait une fonction à la fois d'instruction et réhabilitation. Au début du XVIII ${ }^{\mathrm{e}}$ siècle, le savant et académicien René Antoine Ferchault de Réaumur se lança dans un projet de réforme de l'industrie métallurgique française ${ }^{35}$. Ses recherches et ses premiers dessins servirent de fondation à L'art du serrurier publié en 1767 par l'académicien Henri-Louis Duhamel du Monceau dans le cadre de la Description des arts et métiers édité entre 1761 et 1788 par l'Académie des sciences ${ }^{36}$. La fabrication d'objets ornementaux exigeait des ouvriers à la fois une certaine force physique, mais aussi une capacité à savoir utiliser plusieurs dizaines d'outils (ill. 8). On pouvait fixer sur une enclume des

35. René Antoine Ferchault de Réaumur a publié ses études datées des années 1710 dans L'Art de Convertir le Fer Forgé en Acier et l'Art d'Adoucir le Fer Fondu, Paris, Chez Michel Brunet, 1722, $568 \mathrm{p}$.

36. Henri-Louis Duhamel du Monceau, Art du serrurier, Paris, Chez Saillant \& Nyon et chez Desaint, 1757, $302 \mathrm{p}$. 
étampes rainurées de formes décoratives variées, comme on peut le voir dans les figures 14, 15 et 16. Les ouvriers enfonçaient dessus les barreaux de fer chauffé pour réaliser un motif. Sur cette planche, la représentation de l'objet et la technique ont été réunies dans un but didactique, et le savoir-faire est présenté comme le résultat à la fois du génie créatif et de l'expertise artisanale.

Les planches transmettent un discours selon lequel l'expérience est fondamentale à la compréhension de la technique. Bien que la Description ait reçu l'approbation de l'Académie des sciences, c'est l'Encyclopédie ou Dictionnaire raisonné des sciences, des arts et des métiers, le projet indépendant lancé par Denis Diderot et Jean Le Rond d'Alembert entre 1751 et 1772, qui offre la représentation la plus substantielle du travail au XVIII ${ }^{\mathrm{e}}$ siècle (ill. 9) ${ }^{37}$. Par exemple, une planche en deux parties illustrant la boutique d'un maître-serrurier, montre en-dessous un catalogue indexé des barreaux de fer forgé et en partie supérieure une vignette de leur production. Analysées par Roland Barthes comme les illustrations idéales et rationnelles du travail, ces images visent à expliquer et à systématiser des pratiques qui ont lieu au-delà de la sphère intellectuelle ${ }^{38}$. Si beaucoup a été écrit sur le conflit et les accusations de plagiat entre Réaumur et les contributeurs de l'Encyclopédie, il est important de souligner qu'au milieu du XVIII ${ }^{\mathrm{e}}$ siècle la représentation des techniques de fabrication du fer allait de concert avec une représentation des formes artistique du matériel.

Il convient de rappeler que Jousse a aussi représenté les outils et les techniques de la fabrication du fer, même si son traité n'aspirait pas à la rationalisation $d u$ métier, mais plutôt à l'élévation d'une technique à travers la publication de ses secrets. Le fer constitue pour l'historien un matériau privilégié d'analyse du climat économique et politique, en raison de ses rapports avec les problématiques liées au commerce, à l'industrie de guerre et à l'industrie. En formant les entrelacs d'acanthe et les rainures d'un barreau, les artistes et les artisans ont tenté de mieux comprendre l'art et la science de la serrurerie. La manière dont ils choisissaient de représenter cet art était décidément en dialogue constant et complexe avec la théorie et la pratique de l'architecture en France sous l'Ancien Régime.

Jason NGUYEN

doctorant en histoire de l'architecture, Harvard University

37. Georges Huard, "Les planches de l'Encyclopédie et celles de la Description des Arts et Métiers de l'Académie des Sciences", Revue d'histoire des sciences et leurs applications, t. IV, $\mathrm{n}^{\circ}$ 3-4, 1951, p. 238-249 et plus récemment David Pullins, "Techniques of the Body: Viewing the Arts and Métiers of France from the Workshop of Nicolas I and Nicolas II de Larmessin ", Oxford Art Journal, t. 37, no 2, 2014, p. 135-155.

38. Roland Barthes, "Les planches de l'Encyclopédie ", Le Degré zéro de l'écriture. Nouveaux essais critiques, Paris, Éd. du Seuil, 1972, p. 89-104. 


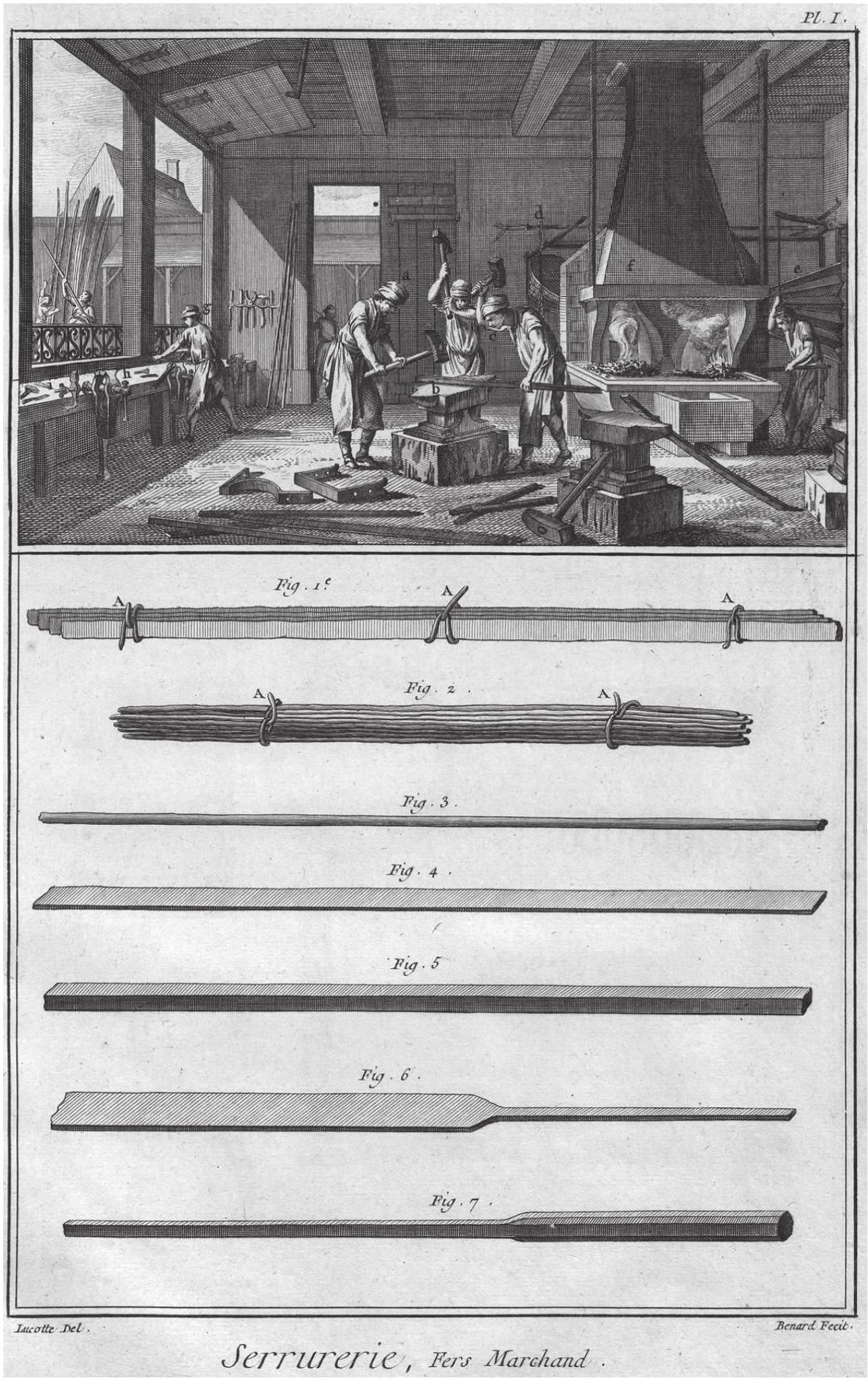

Ill. 9: "Serrurerie, Fers Marchand", gravure à l'eau-forte, Robert Bénard après Jacques-Raymond Lucotte, Recueil des planches, sur les sciences et les arts libéraux, et les arts mécaniques, avec leur explication: Serrurerie, Paris, Chez Briasson, vol. 9, 1771, pl. 1. (C) Cl. Harvard University. 